\title{
Subject Index to Volume 27
}

Acetylcholine, vasodilator action, endothelium-derived relaxing factor as mediator of, guinea pig, 128

$\mathrm{N}$-Acetylglutamate, hepatic and intestinal tissue content, normal and fasted mice, 408

Acidemia

glutaric, type II, phenotype heterogeneity, 311

methylmalonic, urinary metabolite excretion during fasting in children with, 413

propionic, urinary metabolite excretion during fasting in children with, 413

Adolescents, energy expenditure, obese and nonobese subjects, 198

Adrenal gland, calcium homeostasis and, rat, 571

Age, antibody to erythrogenic toxin related to, Kawasaki disease, 11

Airway, pressure, amplitude minimization, during high frequency oscillatory ventilation, rabbit, 64

Allopurinol, hypoxic-ischemic brain injury reduced by, newborn rat, 332

American Pediatric Society

centennial history, S4

presidential address, $\mathrm{S} 1$

Amrinone, biphasic effect, myocardium, neonatal rabbit, 144

Aorta, vascular relaxation, ontogeny of sodium nitroprusside and atrial natriuretic factor responses, guinea pig, 392

Apnea, idiopathic, periodic breathing and, premature infant, 118

Asphyxia, cerebral hemodynamics after, blood glucose concentration effect, neonatal lamb, 454

Atrial natriuretic factor

ontogeny of relaxation response, comparison, atrial natriuretic factor, guinea pig aorta, 392

plasma concentrations, correlation with patent ductus arteriosus shunting, preterm infants with respiratory distress syndrome, 137

water homeostasis and, bronchopulmonary dysplasia, 260

Atrial natriuretic peptide

effect on embryonic hemodynamics, stage 21 chick embryo, 557

exogenous, pulmonary vascular effects, sheep fetus, 140

role in blood volume expansion, weanling rat, 396

Autonomic nervous system, heart rate variation regulated by, neonatal lamb, 383

Bacteria, carbohydrate fermentation patterns, formula-fed and breast-fed infants, 165

Baroreflex, heart rate control, development, piglet, 148

Barotrauma, effect on pulmonary clearance of ${ }^{99 \mathrm{~m}}$ technetium diethylenetriamine pentaacetate, lamb, 70

Bicarbonate, elevated plasma concentration, effect on segmental nephron $\mathrm{HCO}_{3}$ reabsorption, newborn dog, 604
Biliary atresia, extrahepatic, 25-hydroxyvitamin D absorption, 26

Bilirubin

as growth inhibitor, chicken embryo in ovo, 617

blood-brain barrier permeability, injection and perfusion techniques, 436

Biology, developmental, as preventive medicine for neonatology, S21

Blood-brain barrier, permeability, injected and perfused bilirubin, rat, 436

Blood cell, peripheral mononuclear, polymerase activity, Kawasaki disease, 109

Blood flow

cerebral

$\mathrm{CO}_{2}$-related changes, ventilated preterm neonates, 445

perinatal hypoxic-ischemic brain damage, 450

ventilatory response to hypoxia related to, newborn piglet, 327

embryonic, atrial natriuretic peptide effect, chick, 557

pulmonary

atrial natriuretic peptide effect, fetal sheep, 140

changes, during birth-related events, fetal sheep, 372

pulmonary arterial, role, fetal lung growth, sheep, 122

scalp, measurement techniques, lamb, 442

Blood gas, exchange, during high frequency oscillatory ventilation, rabbit, 64

Blood volume, expansion, atrial natriuretic peptide and, weanling rat, 396

Body water, measurement, bioelectrical impedance method, normal and malnourished children, 98

Bone marrow, new human tumorigenic neuroblastoma cell line from, 1

Bradykinin, vasodilator action, endotheliumderived relaxing factor as mediator of guinea pig, 128

Brain

development, docosahexaenoic acid role, chick, 89

hypoxic-ischemic damage

delayed seizures with, fetal sheep, 561

experimental biology of, 317

glucose protective effect, neonatal rat, 186

perinatal, cerebral blood flow and edema in, 450

reduction by allopurinol, newborn rat, 332

injury, prevention, premature infant, $\mathbf{S} 28$

noradrenergic pathways, undernutrition and overnutrition effect, rat, 191

phenylalanine, proton nuclear magnetic resonance observation, rabbit, 566

Brain stem, bilateral lesions, growth stunting and, rat, 181

Breast milk

carbohydrate fermentation, feces comparison, formula-fed infants, 165

glutathione peroxidase activity, impact of gestational length, 32

lactoferrin in stool and urine of infants fed with, 252 selenium concentration, impact of gestational length, 32

Breathing

pattern, analysis, sudden infant death syndrome susceptibility, 113

periodic, idiopathic apnea of prematurity and, 118

Bronchiolitis, water, electrolyte, and endocrine homeostasis in infants with, 204

Bronchopulmonary dysplasia

early stages, changes in vasopressin, atria natriuretic factor and water homeostasis, 260

polymorphonuclear leukocyte superoxide anion release, retinol inhibition, 574

Bronze baby syndrome, animal model for, 22

Calcium, homeostasis, pineal and adrenal effects, rat, 571

Carbamyl phosphate synthetase, deficiency, $\mathrm{N}$-acetylglutamate content in human livers with, 408

Carbohydrate, bacterial fermentation, feces, formula-fed and breast-fed infants, 165

Carbon dioxide

effect on cerebral blood flow, mechanically ventilated preterm neonates, 445

pressure, transcutaneous, scalp blood flow measured by, 442

Cardiomyopathy, hypertrophic, WKY/NCrj rat, 483

Carnitine palmitoyltransferase I and II, defects, immunoquantitative analysis, 497

Cataractogenesis, sugar-induced, STZ-diabetic rat, 293

Cholestasis, chronic, 25-hydroxyvitamin D absorption in, 26

Chondrodysplasia punctata, rhizomelic, aberrant localization of peroxisomal thiolase, 304

Circulation: see Blood flow

Collagen, neonatal platelet response to, 337

Colony stimulating factor, recombinant human granulocyte, group B streptococcal sepsis treatment, neonatal rat, 612

Complement, eighth component, detection of heterozygotes for $\beta$-subunit deficiency, 234

Cooling, evaporative, as adjunct to ice bag use, hyperthermia, primate model, 264

Copper, hepatic metabolism, Menkes' kinky hair syndrome, mouse model, 492

Copper porphyrin, role in bronze baby syndrome, animal model, 22

Cystic fibrosis, Pseudomonas aeruginosa, serum immunoglobulin $G$ antibodies to, 508

Dazmegrel, pretreatment, tumor necrosis factor-induced pulmonary hypertension, 466

Dehydration, diarrheal, treatment, S62

Dehydrogenase, multiple deficiency, lipoic acid effect, infant, 75 
Deoxyribonucleic acid, thymidine incorporation, lactoferrin stimulation, rat crypt enterocytes, 525

Diabetes, proteoglycan metabolism, growth plate, rat, 41

Diabetes mellitus, nonosmotic cataracts, sorbitol-taurine relationship, rat, 293

Diarrhea

acute, trace mineral excretion during, 170 dehydration treatment, American pediatricians, S62

Digoxin, intraamniotic, fetal uptake, sheep, 282

Docosahexaenoic acid, dietary, effect on brain and retina development, chick, 89

Doppler, laser flowmetry, scalp blood flow measured by, lamb, 442

Edema, cerebral, perinatal hypoxic-ischemic brain damage, 450

Electrocorticogram, maturational changes, spectral edge frequency as measure of, fetal lamb, 289

Electrolyte, homeostasis, infants with broncholitis, 204

Embryo

bilirubin and heme as growth inhibitors of chicken embroyos in ovo, 617

hemodynamics, atrial natriuretic peptide effect, chick, 557

Encephalopathy: see Brain

Energy expenditure, obese and nonobese adolescents, 198

Enzyme, antioxidant, prenatal development, rat lung, kidney, heart, 472

Epinephrine, reabsorptive response to, fetal lung liquid, sheep, 588

Erythropoietin, effect on granulocytopoiesis, weanling rat, 583

Escherichia coli, antibody, maternofetal transfer, premature neonate, 365

Eye, nonosmotic cataracts, STZ-diabetic rat, 293

Fatty acid, dietary n-3, nervous tissue development, chick, 89

Fetus

antioxidant enzyme development, rat lung, kidney, heart, 472

bilirubin and heme exposure, effect on growth, chicken embryo, 617

circulation, acute uterine ischemia effect, sheep, 552

development, xanthine oxidase during, 286

hypoxia, met-enkephalin peptide and catecholamine levels, sheep, 52

hypoxic-ischemic encephalopathy, delayed seizures with, sheep, 56

insulin-like growth factor I concentrations, effect of maternal starvation, sheep, 401

intraamniotic digoxin uptake, sheep, 282

intrauterine growth retardation

fetal cardiac blood flow and, 379

hepatic energy and redox states, rat, 56 lung

blood flow, atrial natriuretic peptide effect, sheep, 140

growth, role of pulmonary arterial flow, sheep, 122

lactate metabolism, late gestation alterations, sheep, 274

liquid, reabsorption, triiodothyronine and hydrocortisone effect, sheep, 588

placental transfer of magnesium, rat, 622 protein kinase-C-dependent phosphorylation, rat liver, 599

pulmonary vascular resistance, birth-related changes, sheep, 372

$\mathrm{T}$ and $\mathrm{B}$ lymphocyte populations, distribution, intestine, 239

Follicle-stimulating hormone, pulsatile secretion, immunofluorometric assay, prepubertal and early pubertal boys, 215

Galactose metabolism, ovarian tissue, 117

Galanin, growth hormone secretion stimulated by, somatostatin involvement, 405

Gene, analysis, constitutionally variant stature, 488

Genetic diseases, treatment, case reports, $\mathrm{S} 10$

Genetics

historical events, $\mathrm{S} 17$

molecular, symposium introduction, $\mathrm{S} 8$

Gestation, length, impact on human milk selenium concentration and glutathione peroxidase activity, 32

Glucose

blood concentration, postasphyxia cerebral hemodynamics, neonatal lamb, 454

protective effect, hypoxic brain injury, neonatal rat, 186

Glutamine, transport, maturational changes, rat jejunal brush border membrane vesicles, 519

Glutathione peroxidase, activity, human milk, gestational length and, 32

Granulocytopoiesis, erythropoietin effect, weanling rat, 583

Growth and development

bilirubin and heme effect, chicken embryo, 617

effect of bilateral brain stem lesion, rat, 181

electrocortical activity maturation, spectral edge frequency as measure of, fetal lamb, 289

glutamine transport, maturational changes, jejunal brush border membrane, 519

heart rate, arterial baroreflex control, piglet, 148

intestinal maturation, insulin effect, sucklung ling and weanling rat, 161

role of pulmonary arterial flow, fetal sheep, 122

surfactant palmitic acid clearance, developing and adult rabbit, 268

myocardial diastolic ventricular interaction, pericardial effects, preterm and neonatal lamb, 547

xanthine oxidase activity and substrate affinity, liver, brain, myocardium, intestine, fetal lamb, 286

Growth hormone

galanin-induced secretion, endogenous somatostatin involvement, 405

provocative testing, response prediction by insulin-like growth factor I levels, 45

Growth plate, proteoglycan and cell kinetics, normal, diabetic, and malnourished rats, 41

Growth retardation

intrauterine

fetal hepatic energy and redox states, rat, 56

role of blood flow velocity waveforms

Guanidino, compound analysis, role in hyperargininemia diagnosis, 297
Haemophilus influenzae, type $\mathrm{b}$, functional

Hear activity of antibody to, 358

antioxidant enzyme development, fetal rat, 472

force and oxygen consumption, immature rabbit, 476

ventricle interaction, pericardial effect, preterm and neonatal lamb, 547

Heart rate

arterial baroflex control, developmental course, piglet, 148

variation, regulation by autonomic nervous system, neonatal lamb, 383

Hematopoiesis, regulation, special article, 423

Heme, as growth inhibitor, chicken embryo in ovo, 617

Hemorrhage, maternal, fetal blood flow and, sheep, 552

Heparin, clinical doses, oxygen-induced retinopathy, kitten, 580

Human milk: see Breast milk

Hydrocortisone, synergistic action with triiodothyronine, reabsorption of fetal lung liquid, sheep, 588

25-Hydroxyvitamin D, absorption, chronic childhood cholestasis, 26

Hyperargininemia, diagnosis, role of guanidino compound analysis, 296

Hyperbicarbonemia, euvolemic and hypervolemic, effect on $\mathrm{HCO}_{3}$ reabsorption, newborn dog, 604

Hypertension

pulmonary

group B Streptococcus-induced, piglet, 344

methylprednisolone effect, neonatal lamb, 133

neonatal, dazmegrel pretreatment, 466

Hyperthermia, evaporative cooling as adjunct to ice bag, primate model, 264

Hyperuricemia, in partial hypoxanthineguanine phosphoribosyltransferase deficiency, carrier detection, 417

Hypocalcemia, during phototherapy, pineal and adrenal effects, rat, 571

Hypogonadism, hypogonadotropic, diagnostic methods, 211

Hyponatremia, cerebral edema during, taurine role, cat, 85

Hypoxanthine-guanine phosphoribosyltransferase, partial deficiency, carrier detection, 417

Hypoxia

brain blood flow and ventilatory response to, neonatal piglet, 327

effect on met-enkephalin peptide and catecholamine levels, fetal sheep, 52

perinatal brain damage, cerebral blood flow and edema in, 450

pulmonary vasoconstriction, methylprednisolone effect, neonatal lamb, 133

Imaging, proton nuclear magnetic resonance, phenylalanine, rabbit brain, 566

Immunization, pertussis, metabolic, hematologic, and immunologic functions related to, 353

Immunofluorometry, pulsatile secretion of luteinizing hormone and follicle-stimulating hormone assayed by, prepubertal and early pubertal boys, 215

Immunoglobulin $\mathrm{G}$

serum antibodies, Pseudosmonas aeruginosa, cystic fibrosis, 508

transplacentally acquired antibody, premature neonates, 365 
Immunoglobulin G2, subnormal serum concentrations, healthy children with, 16

Infant: see also Neonate

bronchiolitis, clinical management, 204

human milk fed, lactoferrin in stool and urine of, 252

magnesium absorption and metabolism, measurement, isotopic techniques, 36

medical research, analysis of rejected protocols, 432

multiple dehydrogenase deficiency, lipoic acid effect, 75

preterm

extracellular volume estimation by sucrose dilution, 256

left ventricular output, patent ductus arteriosus, 278

protein-energy malnutrition, refeeding impact after, rabbit, 245

Infant formula

carbohydrate fermentation, feces comparison, breast-fed infants, 165

lactoferrin-supplemented, iron retention from, infant rhesus monkey, 176

Infection, resistance, therapeutic manipulation, S44

Infectious disease

symposium introduction, $\mathrm{S} 38$

100 years perspective, S49

Insulin, effect on small intestine, suckling and weanling rat, 161

Insulin-like growth factor-I

gene analysis, subjects with constitutionally variant stature, 488

plasma concentrations, effect of maternal starvation, ovine fetus, 401

role in predicting growth hormone sufficiency, 45

Interferon- $\alpha$, deficient herpes simplex virusinduced production, blood leukocytes, preterm and term neonates, 7

Interleukin- 6 , production by neonatal leukocytes, 227

Intestine, $\mathrm{T}$ and $\mathrm{B}$ lymphocyte populations, fetal and postnatal tissue samples, 239

Iron, absorption, lactoferrin-supplemented formula, infant rhesus monkey, 176

Ischemia

perinatal brain damage, cerebral blood flow and edema in, 450

uterine, acute, effect on fetal blood flow, sheep, 552

Isotope, stable, magnesium metabolism studied by, full-term infants, 36

Jejunum, brush border membrane, glutamine transport, maturational changes, rat, 519

Kawasaki disease

erythrogenic toxin mitogenicity, age distribution, 11

polymerase activity, cultured peripheral blood mononuclear cells, 109

Kidney

antioxidant enzyme development, fetal rat, 472

hydronephrotic, prostanoid role, 103

Lactate, pulmonary metabolism, late gestation alterations, sheep, 274

Lactoferrin, molecular forms, stool and urine of human milk-fed infants, 252

Learning deficit, after bilateral brain stem lesions, rat, 181

Leukocyte

interferon- $\alpha$ production by, preterm and term neonates, 7 neonatal, interleukin- 6 production by, 227

polymorphonuclear, activation, retinol suppression, 574

Life expectancy, child survival revolution, S56

Lipoic acid, effect in multiple dehydrogenase deficiency, infant, 75

Liver

copper metabolism, mouse model for Menkes' kinky hair syndrome, 492

protein kinase-C phosphorylation, fetal rat, 599

Lung

ntioxidant enzyme development, fetal rat, 472

circulation, changes, during birth-related events, fetal sheep, 372

clearance of surfactant-associated palmitic acid, developing and adult rabbit, 268

fetal development, role of pulmonary arterial flow, sheep, 122

high-frequency jet ventilation effect, newborn piglet, 460

lactate metabolism, late gestation alterations, fetal sheep, 274

surfactant subfraction effect, preterm rabbit, 592

target cells, group B Streptococcus interaction, piglet, 344

${ }^{99 \mathrm{~m}}$ technetium diethylenetriamine pentaacetate clearance, mechanical ventilation and barotrauma effect, lamb, 70

Lung liquid, reabsorption, synergistic action of triiodothyronine and hydrocortisone, fetal sheep, 588

Luteinizing hormone

pulsatile secretion, immunofluorometric assay, prepubertal and early pubertal boys, 215

serum level measurement, boys with delayed puberty, 211

Lymphocyte, distribution, fetal and postnatal intestine, 239

\section{Magnesium}

maternofetal transfer, perfused rat placenta, 622

metabolism, isotopic techniques, full-term infants, 36

Malnutrition

protein-energy, refeeding impact after, infant rabbit, 245

proteoglycan metabolism, growth plate, rat, 41

rotavirus infection with, effect on gut permeability, mice, 153

total body water measurement, bioelectrical impedance method, 98

Menkes' kinky hair syndrome, hepatic copper metabolism, mouse model, 492

Methylprednisolone, effect on hypoxic pulmonary vasoconstriction, neonatal late-gestation lamb, 133

MHC class II, expression, fetal and postnatal intestine, 239

Mineral, excretion, during acute diarrhea treated with oral rehydration, 170

Mortality, child survival revolution, S56

Myocardium, tension development, amrinone effect, neonatal rabbit, 144

Neonate

bronze baby syndrome, animal model for,

diastolic ventricular interaction, pericardial effects, lamb, 547

endothelium-derived relaxing factor, presence, pulmonary and systemic arter- ies, guinea pig, 128

granulocytopoiesis, erythropoietin effect, weanling rat, 583

group B streptococcal sepsis, recombinan human granulocyte colony stimulating factor treatment, rat, 612

heart rate variation, regulation by autonomic nervous system, lamb, 383

high-frequency jet ventilation, early pulmonary changes with, piglet, 460

hypoxic-ischemic brain injury, glucose protective effect, rat, 186

hypoxic pulmonary vasoconstriction, methylprednisolone effect, late-gestation lamb, 133

leukocyte

interferon- $\alpha$ response, term and preterm infants, 7

interleukin- 6 production by, 227

medical progress, S34

myocardium, amrinone effect on tension development, rabbit, 144

nutrition, effect on noradrenergic pathways in brain regions, rat, 191

platelet, deficient collagen-induced activation, 337

Pneumococcus, deficiencies in opsonic defense to, 514

postasphyxia cerebral hemodynamics, blood glucose concentration effect, lamb, 454

premature, placental transfer of Escherichia coli antibody, 365

preventive medicine, role of developmental biology, S21

pulmonary hypertension, dazmegrel pretreatment, 466

red blood cell deformability, rat, 220

segmental nephron $\mathrm{HCO}_{3}$ reabsorption, hyperbicarbonemia effect, dog, 604

symposium introduction, S20

ventilated preterm, carbon dioxide-related changes in cerebral blood flow, 445

ventilatory response to hypoxia, cerebral blood flow and, piglet, 327

Neuroblastoma, new highly tumorigenic human cell line, undetectable N-myc oncogene expression, 1

$\mathrm{N}$-myc oncogene, undetectable expression, new tumorigenic neuroblastoma human cell line with, 1

Nutrition

docosahexaenoic acid role, brain and retina development, chick, 89

noradrenergic development and, neonatal rat, 191

surveillance, total body water measurement by bioelectrical impedance, 98

Obesity, energy expenditure and, adolescent subjects, 198

Ornithine transcarbamylase, deficiency, $\mathrm{N}$ acetylglutamate content in human livers with, 408

Osmoregulation, taurine role, during acute hyponatremia, cat, 85

Ovalbumin, intestinal absorption, malnourished and rotavirus infected mice, 153

Ovary, galactose metabolism, 117

Oxygen

consumption, force and, immature rabbit heart, 476

pressure, transcutaneous, scalp blood flow measured by, 442

Palmitic acid, surfactant-associated, clearance, developing and adult rabbit lung, 268 
Patent ductus arteriosus

shunting, correlation with plasma atrial natriuretic factor concentration, preterm infants with respiratory distress syndrome, 137

stroke volume and left ventricular output, preterm infants, 278

Peptide, met-enkephalin, hypoxia effect, fetal sheep, 52

Pericardium, effect on ventricular coupling, preterm and neonatal lamb, 547

Peroxisome, aberrant localization of thiolase, Zellweger syndrome and rhizomelic chondrodysplasia punctata, 304

Pertussis, immunization, metabolic, hematologic, and immunologic functions related to, 353

Phenotype, heterogeneity, glutaric acidemia type II, 311

Phenylalanine, proton nuclear magnetic resonance observation, rabbit brain, 566

3-Phenylpropionic acid, dehydrogenation, enzymatic basis, 501

Phototherapy, hypocalcemia during, pineal and adrenal effects, rat, 571

Pineal gland, calcium homeostasis and, rat, 571

Placenta

in vitro perfused, taurine transport in, 80

maternofetal transfer of magnesium, rat, 622

Platelet, neonatal, impaired secretion response, 337

Pnemococcus, opsonic defense deficiencies, newborn with adequate complement levels, 514

Polymerase, characterization, cultured blood cells, Kawasaki disease, 109

Pregnancy, acute uterine ischemia, effect on fetal circulation, sheep, 552

Prematurity

brain injury prevention, S28

cerebral blood flow, carbon-dioxide related changes, 445

idiopathic apnea, periodic breathing and 118

lung function, surfactant subfraction effect, rabbit, 592

respiratory distress syndrome, correlation, patent ductus arteriosus shunting with plasma atrial natriuretic factor concentration, 137

retinopathy of, heparin effect, kitten, 580

Primitive culture, paradoxes of aspiration for and of children, S59

Propionate, metabolism disorder, urinary metabolite excretion during fasting in children with, 413

Prostaglandin $\mathrm{E}_{2}$, renal biosynthesis, congenital obstructive uropathy, 103

Prostanoids, renal biosynthesis, congenital obstructive uropathy, 103

Protein, antigen, intestinal uptake, malnourished rotavirus-infected mice, 153

Protein kinase-C, role in growth and metabolic differentiation, fetal rat liver, 599

Proteoglycan, metabolism, growth plate, normal, diabetic, and malnourished rat, 41

Pseudomonas aeruginosa, lipopolysaccharides of, serum immunoglobulin $\mathrm{G}$ antibodies to, cystic fibrosis, 508

Puberty

constitutional delay, luteinizing hormone measurements in boys with, 211

pulsatile secretion of luteinizing hormone and follicle-stimulating hormone, immunofluorometric assay, 215

Pulmonary artery, ligation, fetal lung growth and, sheep, 122

Pulmonary valve, stenosis, WKY/NCrj rat, 483

Red blood cell, morphology and filterability, neonatal and adult rats, 220

Refeeding, impact on intestinal development, after protein-energy malnutrition, infant rabbit, 245

Rehydration, oral therapy, mineral excretion in diarrhea treated with, 170

Relaxing factor, endothelium-derived, neonatal guinea pig, 128

Respiratory distress syndrome, patent ductus arteriosus shunting, correlation with plasma atrial natriuretic factor, preterm infant, 137

Retina

development, docosahexaenoic acid role, chick, 89

oxygen-induced damage, heparin effect, kitten, 580

Retinol, concentration-dependent inhibition, activated neutrophil superoxide anion release, 574

Rotavirus, malnutrition with, effect on intestinal uptake of protein antigen, mice, 153

Seizure, after hypoxic-ischemic encephalopathy, fetal sheep, 561

Selenium, concentration, human milk, gestational length and, 32

Sepsis, group B streptococcal, recombinant human granulocyte colony stimulating factor treatment, neonatal rat, 612

Small intestine

hormonal regulation, suckling and weanling rat, 161

refeeding impact, after protein-energy malnutrition, infant rabbit, 245

Sodium nitroprusside, ontogeny of relaxation response, comparison, atrial natriuretic factor, guinea pig aorta, 392

Somatostatin, endogenous, role in galanininduced growth hormone secretion, 405

Spectral edge frequency, as measure of maturation of electrocortical activity, fetal lamb, 289

Stature, constitutionally variant, insulin-like growth factor-I gene analysis, 488

Streptococcus

group B

organ-specific disposition, piglet, 344

role in oxygen radical-dependent thromboxane release, piglet, 349

sepsis, recombinant human granulocyte colony stimulating factor treatment, neonatal rat, 612

Stroke volume, preterm infants, patient ductus arteriosus, 278

Sucrose, dilution, extracellular volume estimated by, preterm infants, children, and adults, 256

Sudden infant death syndrome, susceptibility, prediction, term infants, 113

Surfactant, subfraction, effect on preterm rabbit lung function, 592

Tacharrhythmia, fetal, intraamniotic digoxin administration, sheep, 282
Taurine

role in osmoregulation, during acute hyponatremia, cat, 85

transport, in vitro perfused human pla centa, 80

${ }^{99} \mathrm{~m}$ Technetium diethylenetriamine pentaacetate, pulmonary clearance, mechanical ventilation and barotrauma effect, lamb, 70

Tetralogy of Fallot, WKY/NCrj rat, 483

Thiolase, peroxisomal, aberrant localization, Zellweger syndrome, 304

Thromboxane, oxygen radical-dependent accumulation, role of Streptococcus group B, piglet, 349

Thromboxane $\mathrm{B}_{2}$, renal biosynthesis, congenital obstructive uropathy, 103

Thymidine, incorporation into DNA, lactoferrin stimulation, rat crypt enterocytes, 525

Toxin, erythrogenic, measurement of serum antibody against, 11

Triiodothyronine, synergistic action with hydrocortisone, reabsorption of fetal lung liquid, sheep, 588

Tumor necrosis factor, neonatal pulmonary hypertension induced by, dazmegrel pretreatment, 466

Uropathy, congenital obstructive, prostanoid role, 103

Vascular resistance, pulmonary, birth-related changes, fetal sheep, 372

Vasoconstriction, pulmonary, methylprednisolone effect, neonatal lamb, 133

Vasopressin, water homeostasis and, bronchopulmonary dysplasia, 260

Ventilation

high-frequency jet, early pulmonary changes with, neonatal piglet, 460

high frequency oscillatory, adequate gas exchange and low alveolar pressure during, rabbit, 64

mechanical

carbon dioxide-related changes in cerebral blood flow, preterm neonate, 445 effect on pulmonary clearance of ${ }^{99} \mathrm{~m}$ technetium diethylenetriamine pentaacetate, lamb, 70

oscillatory patterns, sudden infant death syndrome, 113

Ventricular septal defect, WKY/NCrj rat, 483

Viral disease, control, past successes, S39

Volume, extracellular, estimation by sucrose dilution, preterm infants, children and adults, 256

Water

homeostasis

infants with bronchiolitis, 204

vasopressin and atrial natriuretic factor and, bronchopulmonary dysplasia, 260

World's children symposium, introduction, S55

Xanthine oxidase, activity and substrate affinity, during human fetal development, 286

Zellweger syndrome, peroxisomal thiolase in, aberrant localization, 304 